\title{
The contribution of bacteroidal nitrate and nitrite reduction to the formation of nitrosylleghaemoglobin complexes in soybean root nodules
}

\author{
Georgina E. Meakin, ${ }^{1}$ Emilio Bueno, ${ }^{2}$ Brian Jepson, ${ }^{1}$ Eulogio J. Bedmar, ${ }^{2}$ \\ David J. Richardson ${ }^{1}$ and María J. Delgado ${ }^{2}$ \\ ${ }^{1}$ School of Biological Sciences, University of East Anglia, Norwich NR4 7TJ, UK \\ ²Estación Experimental del Zaidín, CSIC, PO Box 419, 18080 Granada, Spain
}

Correspondence

Georgina E. Meakin

g.meakin@uea.ac.uk

Received 13 July 2006

Revised 10 November 2006

Accepted 13 November 2006
It is becoming recognized that leghaemoglobin constitutes an important buffer for the cytotoxic nitric oxide radical $\left(\mathrm{NO}^{\circ}\right)$ in root nodules, although the sources of this $\mathrm{NO}^{\circ}$ within nodules are unclear. In Bradyrhizobium japonicum bacteroids, $\mathrm{NO}^{\circ}$ can be produced through the denitrification process, during which nitrate is reduced to nitrite by the periplasmic nitrate reductase Nap, and nitrite is reduced to $\mathrm{NO}^{\circ}$ by the respiratory nitrite reductase NirK. To assess the contribution of bacteroidal denitrification to the $\mathrm{NO}^{*}$ within nitrate-treated soybean nodules, electron paramagnetic resonance and UV-visible spectroscopy were employed to study the presence of nitrosylleghaemoglobin (LbNO) within nodules from plants inoculated with wild-type, napA or nirK $B$. japonicum strains. Since it has been found that hypoxia induces NO production in plant root tissue, and that plant roots can be subjected to hypoxic stress during drought and flooding, the effect of hypoxic stress on the formation of LbNO complexes within nodules was also investigated. Maximal levels of LbNO were observed in nodules from plants treated with nitrate and subjected to hypoxic conditions. It is shown that, in the presence of nitrate, all of the LbNO within normoxic nodules arises from nitrate reduction by the bacteroidal periplasmic nitrate reductase, whereas Nap activity is only responsible for half of the LbNO within hypoxic nodules. In contrast to Nap, NirK is not essential for LbNO formation under any condition tested.

\section{INTRODUCTION}

Bradyrhizobium japonicum is a Gram-negative soil $\alpha$-proteobacterium that has the ability to form dinitrogenfixing symbiotic nodules with soybean plants (Glycine max). Within these nodules, vegetative cells differentiate into bacteroids, which then synthesize nitrogenase, the enzyme responsible for the reduction of dinitrogen to ammonia. Nitrogenase requires both a low partial oxygen pressure and a high dose of ATP for its activity. These conflicting demands are met by the control of oxygen flux through a diffusion barrier in the inner cortex of the nodules (Minchin, 1997), and by leghaemoglobin (Lb), a highaffinity carrier protein, which binds oxygen and delivers it to the bacteroids (Appleby, 1992). To cope with the micro-oxic conditions (3-22 $\mathrm{nM} \mathrm{pO}_{2}$ ) prevailing in the infected plant tissue, dinitrogen-fixing bacteroids use a $c b b_{3}$-type high-affinity terminal cytochrome oxidase

Abbreviations: EPR, electron paramagnetic resonance; Lb, leghaemoglobin; $\mathrm{Lb}^{2+}$, ferrous leghaemoglobin; Lba, soybean leghaemoglobin a; $\mathrm{LbNO}$, nitrosylleghaemoglobin; $\mathrm{LbO}_{2}$, oxyleghaemoglobin; MV, methyl viologen; NOS, nitric oxide synthase; WT, wild-type. encoded by the fixNOQP operon (Delgado et al., 1998; Preisig et al., 1996).

Nitric oxide $\left(\mathrm{NO}^{\circ}\right)$ is both a gaseous free radical and a versatile cell-signalling effector that plays important roles in diverse (patho)physiological processes in plants (Lamotte et al., 2005). In nodules, $\mathrm{NO}^{*}$ is produced (Baudouin et al., 2006) and can bind $\mathrm{Lb}$ to form nitrosylleghaemoglobin (LbNO) complexes, the presence of which has been observed within nodules using UV-visible (Kanayama et al., 1990; Kanayama \& Yamamoto, 1990a) and electron paramagnetic resonance (EPR) (Mathieu et al., 1998) spectroscopy. Recently, it has been proposed that oxyleghaemoglobin $\left(\mathrm{LbO}_{2}\right)$ is able to scavenge any $\mathrm{NO}^{\circ}$ formed in functional nodules (Herold \& Puppo, 2005), contributing to the protection of nitrogenase, which is rapidly inactivated by this reactive species. However, although the formation of LbNO complexes has been demonstrated in nodules, the sources of the $\mathrm{NO}^{\circ}$ in these complexes are still unclear.

In nodules, $\mathrm{NO}^{\bullet}$ may originate from the plant tissue or from the bacteroids. Two possible sources of $\mathrm{NO}^{\bullet}$ in plant 
tissue are nitrite and arginine. The plant nitrate reductase (Yamasaki \& Sakihama, 2000; Yamasaki et al., 1999) and root mitochondria (Gupta et al., 2005) have been shown to reduce nitrite to $\mathrm{NO}^{\circ}$, although whether these processes have a role in $\mathrm{NO}^{\circ}$ production in nodules has yet to be addressed. $\mathrm{NO}^{\circ}$ may also be produced in plant tissue from arginine by a nitric oxide synthase (NOS), the activity of which has been identified in the nodules of Lupinus albus (Cueto et al., 1996), and the gene for which, AtNOS1, has been identified in Arabidopsis thaliana (Guo et al., 2003). Whether AtNOS1 is present in soybean nodules is unknown.

In B. japonicum bacteroids, denitrification, the sequential reduction of nitrate or nitrite to dinitrogen via the intermediate compounds $\mathrm{NO}^{\circ}$ and nitrous oxide, is a source of $\mathrm{NO}^{\circ}$. In B. japonicum, denitrification depends on the napEDABC (Delgado et al., 2003), nirK (Velasco et al., 2001), norCBQD (Mesa et al., 2002) and nosRZDYFLX (Velasco et al., 2004) genes, encoding nitrate-, nitrite-, nitric oxide- and nitrous oxide-reductase enzymes, respectively. Expression of B. japonicum denitrification genes has been reported in isolated bacteroids and in soybean root nodules by in situ histochemical detection of $\beta$-galactosidase activity from transcriptional fusions of the nirK, nor C and nosZ genes to the reporter gene lacZ (Mesa et al., 2004). If the nap genes are also expressed within bacteroids, then the reduction of nitrate to $\mathrm{NO}^{\circ}$ by Nap and NirK may be a possible route for $\mathrm{NO}^{\circ}$ production within nodules. However, nitrate and nitrite metabolism in nodules could be very complex, as the B. japonicum genome has genes that potentially encode cytoplasmic nitrate (nasA) and nitrite reductases (nirB; Kaneko et al., 2002). The gene products of nas $A$ and nirB are potentially involved in nitrate/nitrite assimilation, but the regulation of the expression and activity of the NasA and NirB enzymes is currently unknown.

Recently it has been found that $\mathrm{NO}^{\circ}$ is produced in plant root tissue when it is subjected to hypoxic stress (Dordas et al., 2003, 2004). It has been proposed that nitrate reduction by the plant nitrate reductase is responsible for this hypoxia-induced $\mathrm{NO}^{*}$ increase, as the presence of nitrate has been shown to enhance $\mathrm{NO}^{\circ}$ production in response to hypoxic stress (Dordas et al., 2004). Since plant roots can be subjected to hypoxic stress during drought and flooding, the latter causing waterlogging of the soil that can be a particular problem for soybean crops (Linkemer et al., 1998), hypoxia may cause an increase in LbNO formation within soybean root nodules. If this were to occur, and since there is a bacteroidal component in nodules, then nitrate and nitrite reduction by the bacteroids may contribute to hypoxia-induced $\mathrm{NO}^{\circ}$ production. This has been assessed in the present study by using EPR and UV-visible spectroscopy to investigate the presence of LbNO within nodules from plants inoculated with the wild-type (WT), napA (Delgado et al., 2003) or nirK (Velasco et al., 2001) B. japonicum strains.

\section{METHODS}

Bacterial strains, plasmids and growth conditions. B. japonicum USDA110 (US Department of Agriculture, Beltsville, MD), and napA GRPA1 (Delgado et al., 2003) and nirK GRK308 (Velasco et al., 2001) mutant derivative strains were used in this study. B. japonicum strains 0602 and 2498 are USDA110 derivatives that contain a chromosomally integrated transcriptional lac $Z$ fusion to the promoter region of the napEDABC genes (Robles et al., 2006) or nirK gene (Mesa et al., 2003), respectively, which have been used to monitor nap and nirK expression in bacteroids. B. japonicum strains were routinely grown in peptone-salts-yeast extract (PSY) medium (Regensburger \& Hennecke, 1983) at $28^{\circ} \mathrm{C}$. Antibiotics were added to B. japonicum cultures at the following concentrations $\left(\mu \mathrm{g} \mathrm{ml}^{-1}\right)$ : chloramphenicol, 15; spectinomycin, 200; streptomycin, 200; tetracycline, 100 .

Plant growth conditions. Soybean (Glycine max L. Merr., cv. Williams) seeds were surface-sterilized with $96 \%$ ethanol (v/v) for $30 \mathrm{~s}$, immersed in $\mathrm{H}_{2} \mathrm{O}_{2}(15 \%, \mathrm{v} / \mathrm{v})$ for $8 \mathrm{~min}$, then washed thoroughly with sterile water and germinated in darkness at $28{ }^{\circ} \mathrm{C}$. Selected seedlings were planted in autoclaved 11 Leonard jar assemblies filled with vermiculite. Plants (two per jar) were inoculated at sowing with $1 \mathrm{ml}$ of a single bacterial strain $\left(10^{8} \mathrm{cells}^{-1}\right)$, supplied with a nitrogen-free mineral solution (Rigaud \& Puppo, 1975), and grown in a controlled environmental chamber under conditions previously described (Delgado et al., 1998). After growth for 28 days, one set of plants was provided with the same mineral solution supplemented with $4 \mathrm{mM} \mathrm{KNO}_{3}$, while the other set was maintained solely under $\mathrm{N}_{2}$-fixing conditions. A nitrate concentration of $4 \mathrm{mM}$ was used, as it has previously been demonstrated that although treatment of plants with $4 \mathrm{mM} \mathrm{KNO}_{3}$ does result in denitrification activity, it does not inhibit nodule formation or nitrogenase activity (Mesa et al., 2004). Seven days before nodule harvesting, a set of nitrate-treated plants and a control set of untreated plants were subjected to hypoxia by transferring 33-day-old plants from the Leonard jars into bottles containing nutrient solution. The bottles were bunged with cotton wool and made hypoxic by sparging with argon for $15 \mathrm{~min}$, and then silicone was spread on top of the cotton wool to seal the bottles. Another two sets of control plants and nitrate-treated plants were maintained under atmospheric conditions for one additional week before nodule harvesting.

Bacteroid isolation. Nodules were harvested from 40-day-old plants and bacteroids were prepared as previously described (Mesa et al., 2004). In brief, $1.5 \mathrm{~g}$ of fresh nodules was ground in $7.5 \mathrm{ml}$ Tris/ $\mathrm{HCl}$ ( $\mathrm{pH} 7.5$ ) supplemented with $250 \mathrm{mM}$ mannitol. The homogenate was filtered through four layers of cheesecloth and centrifuged at $250 \mathrm{~g}$ at $4{ }^{\circ} \mathrm{C}$ for $5 \mathrm{~min}$ to remove nodule debris. The resulting supernatant was recentrifuged at $12000 \mathrm{~g}$ at $4{ }^{\circ} \mathrm{C}$ for $10 \mathrm{~min}$ to pellet the bacteroids. The bacteroids were washed twice with $50 \mathrm{mM}$ Tris/ $\mathrm{HCl}(\mathrm{pH} 7.5)$ and resuspended in a final volume of $2.5 \mathrm{ml}$.

Analytical methods. For determination of $\beta$-galactosidase activity, bacteroids were resuspended in $100 \mathrm{mM}$ sodium phosphate buffer ( $\mathrm{pH} 7.0$ ) to an $\mathrm{OD}_{600}$ of $\sim 0.6$. $\beta$-Galactosidase activities were determined from permeabilized bacteroids from at least three independent preparations, as described previously (Miller, 1972). Bacteroids were not kept micro-oxic but were used immediately for assays. All media and materials used for incubation were sterilized at $120 \mathrm{kPa}$ and $110^{\circ} \mathrm{C}$ for $30 \mathrm{~min}$ before use.

Methyl viologen (MV)-dependent nitrate reductase and nitrite reductase activities were analysed essentially as described by Delgado et al. (2003). Nitrite concentration was estimated after diazotization by adding the sulfanilamide/naphthylethylene diamine dyhydrochloride reagent (Nicholas \& Nason, 1957). 
The protein concentration of bacteroids was estimated by using the Bio-Rad assay, with a standard curve of varying BSA concentrations.

For determination of nitrite accumulation in nodules, $0.5 \mathrm{~g}$ of nodules was homogenized with $2 \mathrm{ml} 1 \mathrm{M}$ zinc acetate and centrifuged at $12000 \mathrm{~g}$ at $4{ }^{\circ} \mathrm{C}$ for $5 \mathrm{~min}$. The resulting supernatant was mixed with 1 vol. $100 \%$ ice-cold ethanol and centrifuged for a further $5 \mathrm{~min}$. The nitrite concentration of the final supernatant was determined as described above.

Whole-nodule EPR spectroscopy. Whole-nodule EPR spectroscopy was performed essentially as described by Mathieu et al. (1998). Intact frozen nodules were transferred directly to cylindrical EPR tubes to a depth of approximately $4 \mathrm{~cm}$. Although attempts were made to load nodules of similar size to the tubes, the actual amount of tissue within each tube varied within a maximum of $30 \%$. EPR spectra were recorded at $60 \mathrm{~K}$ on a Bruker EMX system $\mathrm{X}$-band spectrometer equipped with an Oxford Instruments ESR-9 liquid helium flow cryostat. The instrument settings were as follows: microwave power $2 \mathrm{~mW}$, modulation amplitude $10 \mathrm{G}$, modulation frequency $100 \mathrm{kHz}$, microwave frequency $9.67 \mathrm{GHz}$ and magnetic field scan range from 3000 to $3800 \mathrm{G}$.

UV-visible spectroscopy. For the quantification of the percentage of $\mathrm{Lb}$ bound to $\mathrm{NO}^{\circ}$ within nodules, the procedure was as follows. First, Lb extracts were prepared from nodules by homogenizing $0.3 \mathrm{~g}$ of nodules with $4 \mathrm{ml} \mathrm{Lb}$ extraction buffer $[40 \mathrm{mM}$ $\mathrm{Na}_{2} \mathrm{HPO}_{4} .2 \mathrm{H}_{2} \mathrm{O}(\mathrm{pH} 7.4) ; 10 \mathrm{mM} \mathrm{NaH} \mathrm{PO}_{4} \cdot \mathrm{H}_{2} \mathrm{O}(\mathrm{pH} 7.4) ; 0.02 \%$ $\mathrm{K}_{3} \mathrm{Fe}(\mathrm{CN})_{6} ; 0.1 \% \mathrm{NaHCO}_{3}$, centrifuging at $12000 \mathrm{~g}$ at $4{ }^{\circ} \mathrm{C}$ for $20 \mathrm{~min}$ and retaining the supernatant. As a control, soybean leghaemoglobin $a(\mathrm{Lb} a)$ was purified from an overexpressing E. coli strain, as described by Jones et al. (1998). Second, since Lb directly extracted from nodules was a mixture of LbNO, ferric leghaemoglobin $\left(\mathrm{Lb}^{3+}\right)$ and $\mathrm{LbO}_{2}$, nodule extracts were reduced by the addition of excess sodium dithionite to convert the $\mathrm{Lb}^{3+}$ and $\mathrm{LbO}_{2}$ to a single species, ferrous leghaemoglobin $\left(\mathrm{Lb}^{2+}\right)$. Samples of reduced $\mathrm{Lb} a$ were also prepared through the addition of excess sodium dithionite. Third, all of the $\mathrm{Lb}$ in the reduced nodule extracts and purified Lba samples was converted to LbNO by the addition of excess nitrite. Next, absorption spectra from 300 to $700 \mathrm{~nm}$ at $20^{\circ} \mathrm{C}$ were recorded from $1 \mathrm{ml}$ reduced and nitrosylated nodule extracts and Lba samples using a Hitachi U-3310 spectrophotometer linked to a circulating BC-10 water bath (Fisher Scientific). Finally, absorption measurements (described below) were taken from the spectra to allow quantification of the nodular LbNO using equation (2) below, derived from the following rationale.

Since the reduced nodule extracts contained only $\mathrm{Lb}^{2+}$, if $\mathrm{LbNO}$ was absent, or a mixture of $\mathrm{Lb}^{2+}$ and $\mathrm{LbNO}$, if LbNO was present, then the difference between the absorption spectra from the reduced and nitrosylated extracts would give an indication of the proportion of LbNO within the nodules. The difference between the reduced and nitrosylated spectra was measured as the difference between the absorption of the two spectra at $579 \mathrm{~nm}\left(\Delta \mathrm{A}_{579}=\mathrm{A}_{579}^{\mathrm{LbNO}}-\mathrm{A}_{579}^{\mathrm{Lb}^{2+}}\right)$, the wavelength at which a maximum difference between the two spectra was observed. This difference was then divided by the absorbance at the isosbestic point at $590 \mathrm{~nm}$ to nullify the effect of differences in concentration between the samples. To quantify these differences, the difference between the reduced and nitrosylated Lba spectra $\left(\Delta A_{579}{ }^{\mathrm{Lb} a}\right)$ was used as a reference for zero LbNO, as shown in equation (1):

(1) $0 \% \mathrm{LbNO}=\Delta A_{579}^{\mathrm{Lba}} / A_{590}^{\mathrm{Lba}}=0.081 / 0.2865=0.28$

The ratios of $\Delta A_{579} / A_{590}$ for the nodule extracts were determined and used in equation (2) to give the percentage of $\mathrm{Lb}^{\circ}$ bound to $\mathrm{NO}^{\circ}$ within the original nodule extracts:

(2) Percentage $\mathrm{LbNO}=\left(0.28-\Delta A_{579} / A_{590}\right) / 0.28$

\section{RESULTS}

\section{LbNO and nitrite accumulation in normoxic WT nodules}

To confirm the observation of Kanayama and others (Kanayama et al., 1990; Kanayama \& Yamamoto, 1990a) that LbNO is present within nodules from nitrate-treated plants, spectroscopic techniques were performed on nodules harvested from soybean plants inoculated with the WT $B$. japonicum strain and supplemented with $4 \mathrm{mM}$ nitrate. Whole-nodule EPR spectroscopy was used to detect LbNO non-invasively, and UV-visible spectroscopy was used to quantify the proportion of $\mathrm{Lb}$ bound to $\mathrm{NO}^{*}$ in nodule extracts (see Methods). An EPR signal corresponding to LbNO was observed within these WT nodules (Fig. la i). Inspection of the absorption spectra from extracts of these nodules (Fig. 2a) showed that approximately $25 \%$ of the $\mathrm{Lb}$ within these nodules was bound to $\mathrm{NO}^{\circ}$ (Fig. 3a). In addition, Kanayama \& Yamamoto (1990a) have proposed that nodular nitrite accumulation is the source of LbNO within nitrate-treated nodules. To check that nitrite was produced within our nitrate-treated nodules, nitrite accumulation was determined within WT normoxic nodules, which revealed that nitrite had accumulated to a level of approximately $230 \mathrm{nmol}$ (g fresh-weight nodule) $)^{-1}$ (Fig. 3b).

\section{Bacteroidal contribution to LbNO formation within normoxic WT nodules}

Nitrite accumulation within nitrate-treated nodules, a possible source of $\mathrm{LbNO}$, has been proposed to arise mainly from the reduction of nitrate by the plant nitrate reductase (Kanayama et al., 1990). However, those authors did not fully explore the possibility that the bacteroids might play a role in this nitrite accumulation and nitrate and nitrite reduction. Since B. japonicum cells can reduce nitrate to nitrite through the action of the periplasmic nitrate reductase Nap (Delgado et al., 2003), and reduce nitrite to $\mathrm{NO}^{*}$ through the action of the respiratory nitrite reductase NirK (Velasco et al., 2004), the expression of the nap operon and nirK gene was assessed in bacteroids. Plants were inoculated with a $B$. japonicum strain containing a chromosomally integrated $\mathrm{P}_{\text {napE }}-$ lac $Z$ or $\mathrm{P}_{\text {nirK }}-$ lac $Z$ fusion. Bacteroids prepared from nodules of these plants exhibited both nap and nirK expression (Fig. 4a). In addition, bacteroids from WT nodules showed MV-dependent nitrate reductase activity at a rate of $328 \pm 69 \mathrm{nmol} \mathrm{NO}_{2}^{-}$produced $\mathrm{h}^{-1}$ (mg protein) $)^{-1}$ (Fig. $\left.4 \mathrm{~b}\right)$, and nitrite reductase activity at $144 \pm 7 \mathrm{nmol} \mathrm{NO}_{2}^{-}$consumed $\mathrm{h}^{-1}(\mathrm{mg} \text { protein })^{-1}$ (Fig. 4c). 
(a) $\mathrm{i}$

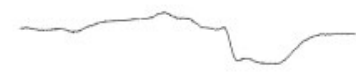

iii

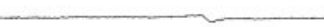

$\mathbf{v}$
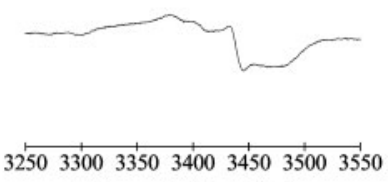
Magnetic field $(\mathrm{G})$

(b)

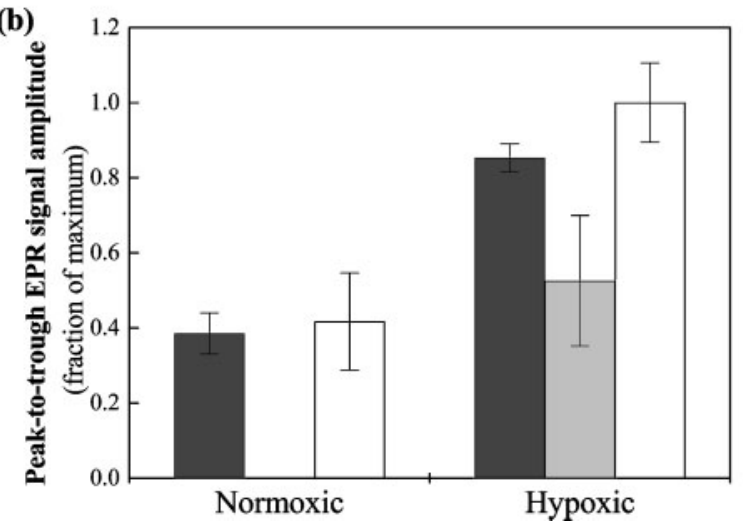

Fig. 1. (a) EPR spectra from intact normoxic $B$. japonicum WT (i), napA (iii) and nirk (v) nodules or hypoxic WT (ii), napA (iv) and nirk (vi) nodules. The $y$ axis has the same scale in i-vi. Spectra are representative of EPR spectra recorded from triplicate nodule samples from two or three different nodule harvests. (b) Peak-to-trough amplitudes (mean \pm SEM), presented as fractions of the maximum EPR signal observed, determined from EPR spectra of WT (black bars), napA (grey bars) and nirk (white bars) nodules from plants treated with nitrate, presented as fractions of the maximum EPR signal observed. Amplitudes were determined from EPR spectra recorded from triplicate nodule samples from at least two different nodule harvests.

\section{Nap activity and LbNO formation in normoxic nodules}

Since the nap operon is expressed in nitrate-treated nodules, then the periplasmic nitrate reductase may have a role in LbNO formation. To investigate this, plants were inoculated with a napA mutant strain and the nodules harvested. To assess the phenotype of this mutant, MV-dependent nitrate reductase activity was measured in bacteroids prepared from the napA nodules. As Fig. 4(b) shows, no nitrate reductase activity was observed in the napA bacteroids. To see whether
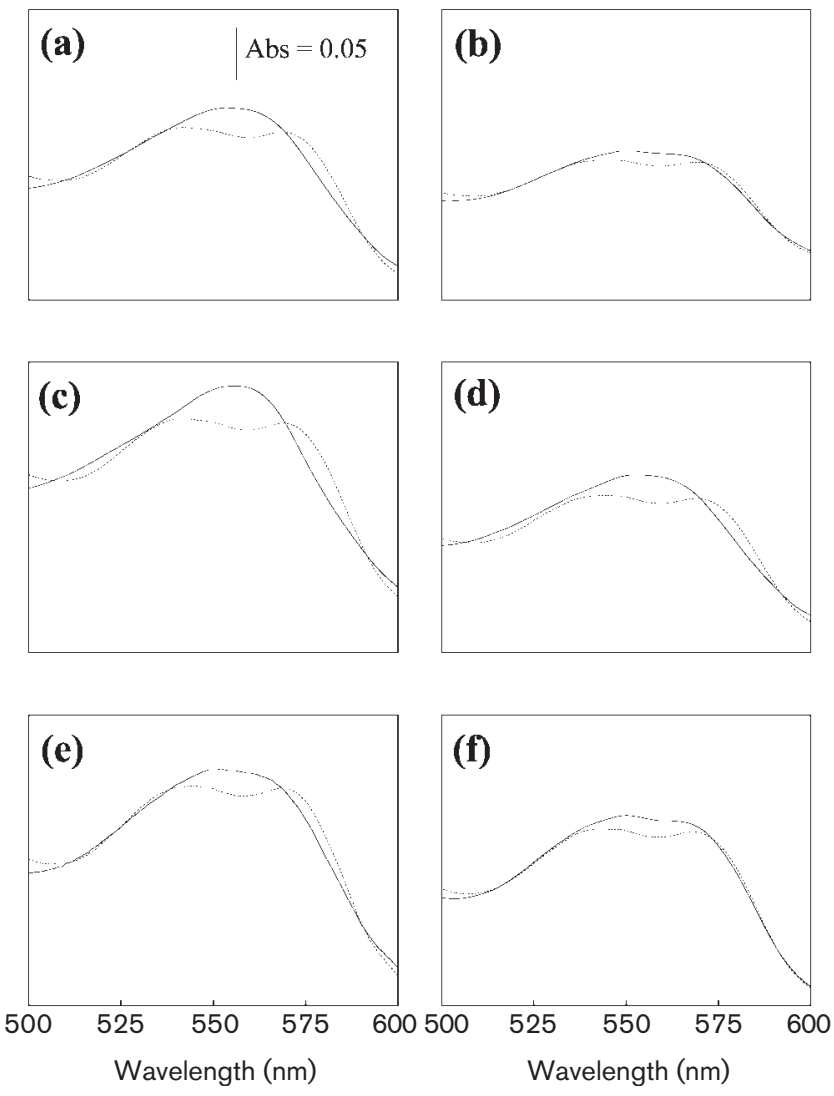

Fig. 2. UV-visible spectra from reduced (solid line) and nitrosylated (dotted line) normoxic B. japonicum WT (a), napA (c) and nirK (e) nodule extracts or hypoxic WT (b), napA (d) and nirk (f) nodule extracts. The absorbance (Abs) scale is indicated in panel (a) and is the same for all panels. Spectra are representative of UV-visible spectra recorded from triplicate nodule extracts from at least two different nodule harvests.

this lack of bacteroidal nitrate reductase activity in the napA nodules affected the level of nitrite accumulation and LbNO within the nodules, nodular nitrite accumulation was determined, and EPR and UV-visible spectra were taken from whole nodules and nodule extracts, respectively. No nitrite accumulation (Fig. 3b) and no LbNO EPR signal was observed within napA nodules, only a signal corresponding to the presence of organic radicals remained in the EPR spectrum (Fig. 1a iii). Consistent with this finding, the difference between the reduced and nitrosylated UV-visible spectra from the napA nodule extract (Fig. 2c) was similar to that observed between the spectra from purified Lba samples (data not shown), demonstrating the lack of LbNO in napA normoxic nodules (Fig. 3a).

\section{NirK activity and LbNO formation in normoxic nodules}

Since we have demonstrated that, in nitrate-treated normoxic nodules, nitrate reduction by NapA is the sole source 

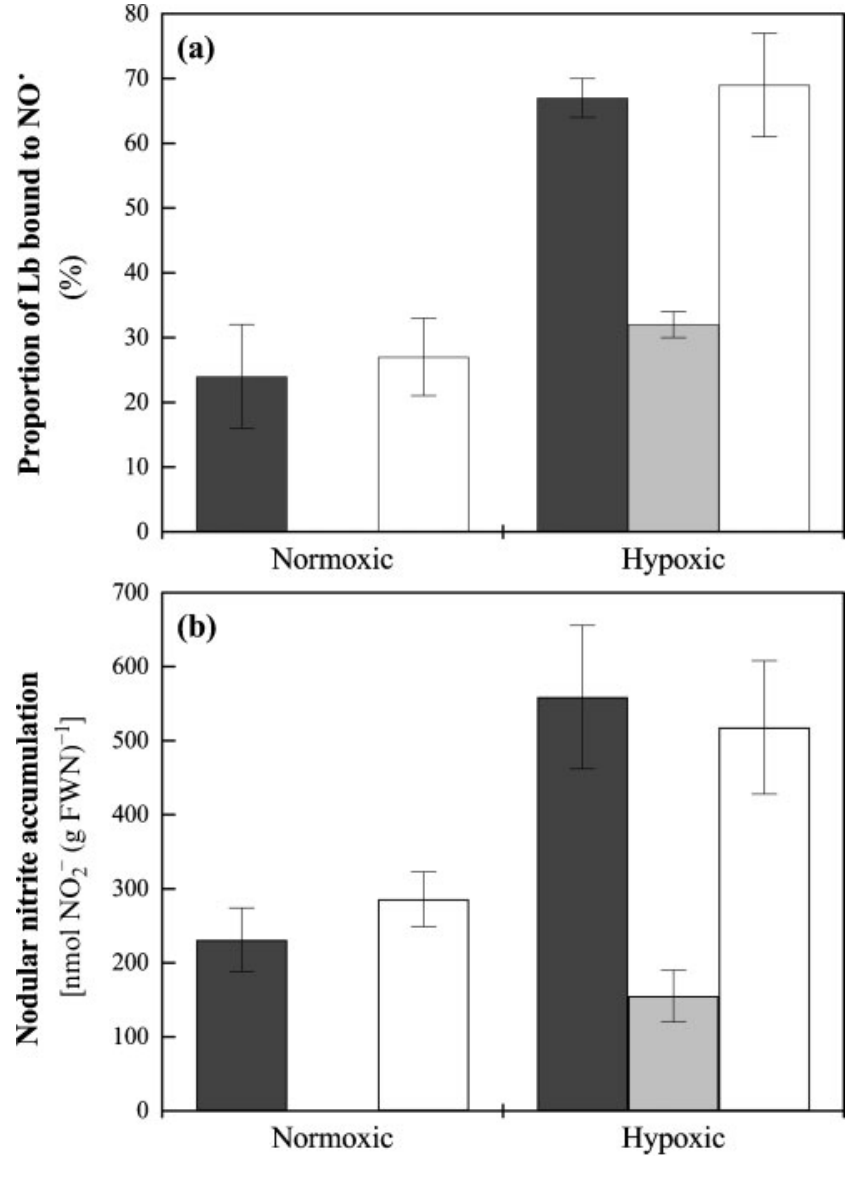

Fig. 3. LbNO proportion (a) and nitrite accumulation (b) within $B$. japonicum WT (black bar), napA (grey bar) and nirK (white bar) nodules. LbNO proportion was determined from the UVvisible spectra presented in Fig. 2, using the calculation described in Methods. Data are means \pm SEM from at least two different nodule harvests, assayed in triplicate. FWN, freshweight nodule.

of LbNO within nodules, then it is possible that the next step in the denitrification pathway, the reduction of nitrite to $\mathrm{NO}^{\circ}$ by NirK, may also play a role in LbNO production. To investigate this, bacteroidal nitrite reductase activity and nodular nitrite and LbNO accumulation were determined in nodules from plants inoculated with a nirK mutant strain. As Figs 4(c) and 3(b) show, similar levels of bacteroidal nitrite reductase activity and nodular nitrite accumulation were observed between the nirK and WT nodules. Observation of the EPR spectra from the WT and nirK nodules (Fig. 1a $\mathrm{i}$ and $\mathrm{v}$ ) showed that similar EPR signal amplitudes were observed in both nodule samples (Fig. 1b). Likewise, similar differences between the reduced and nitrosylated nodule extract absorption spectra were observed between the nirK and WT nodules (Fig. 2a, e), corresponding to approximately $25 \%$ of the $\mathrm{Lb}$ being nitrosylated (Fig. 3a).
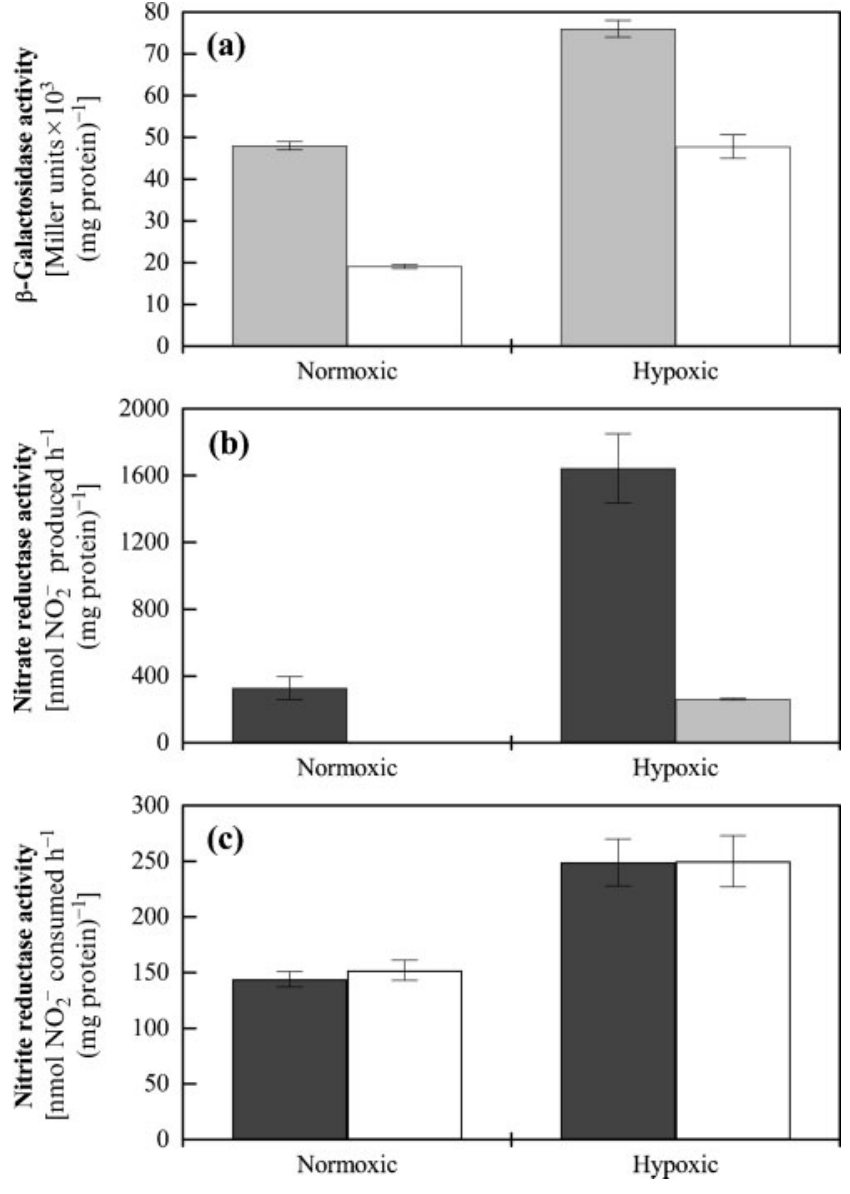

Fig. 4. (a) $\beta$-Galactosidase activity from a $P_{\text {napE }}-$ lac $Z$ (grey bar) or $\mathrm{P}_{\text {nirk }}-\mathrm{lac} Z$ (white bar) fusion in WT $B$. japonicum bacteroids. MV-dependent nitrate reductase (b) or nitrite reductase (c) activity in bacteroids from WT (black bars), napA (grey bars) and nirk (white bars) nodules is shown. Bacteroids were prepared from normoxic and hypoxic nodules, as described in Methods. Data are means \pm SEM from at least two different bacteroid isolations, assayed in triplicate.

\section{Hypoxia, LbNO formation and nitrite accumulation in soybean nodules}

Recent studies have found that $\mathrm{NO}^{\bullet}$ levels in plant tissue are elevated when the tissue is subjected to hypoxic stress (Dordas et al., 2003, 2004). To investigate whether hypoxic stress causes an increase in LbNO formation within soybean root nodules, the roots of soybean plants, inoculated with the WT B. japonicum strain and supplemented with nitrate, were subjected to hypoxic stress, as described in the Methods. The EPR spectra from whole WT nodules demonstrated that hypoxia caused just over a twofold increase in the EPR LbNO signal (Fig. 1a $\mathrm{i}$ and ii, b). The absorption spectra from the hypoxic WT nodule extract (Fig. 2b) showed that the reduced spectrum was similar to the nitrosylated spectrum, suggesting that the original extract contained a higher proportion of LbNO than the 
normoxic WT extract (Fig. 2a). In confirmation of this, interpretation of the absorption spectra found that approximately $70 \%$ of the Lb in the hypoxic WT nodules was bound to $\mathrm{NO}^{\circ}$ (Fig. 3a), 2.5 times that found in the normoxic nodules. Correlated with this, approximately 2.5 times more nitrite accumulated within the hypoxic WT nodules than in the normoxic nodules (Fig. 3b).

\section{Bacteroidal nap and nirK expression and nitrate and nitrite reductase activities following hypoxic stress}

To investigate the possible role of the bacteroidal Nap and NirK enzymes in this hypoxic increase in LbNO formation, the expression levels of the nap operon and nirK gene, and the rates of bacteroidal nitrate and nitrite reductase activities, were determined in WT nodules from plants subjected to hypoxic stress. As Fig. 4(a, b) shows, hypoxia caused nap expression to increase 1.5-fold, although bacteroidal nitrate reductase activity actually underwent a fivefold increase in response to hypoxic stress. Hypoxia also resulted in a twofold increase in nirK expression and nitrite reductase activity in bacteroids (Fig. 4a, c).

\section{The contribution of NapA to LbNO formation in hypoxic nodules}

Since nap expression and nitrate reductase activity were upregulated in bacteroids from nodules of plants subjected to hypoxic stress, then Nap may have a role in the formation of LbNO within hypoxic nodules. To investigate the contribution of nitrate reduction by Nap to LbNO formation in response to hypoxic stress, bacteroidal nitrate reductase activity and nodular nitrite and LbNO accumulation were observed in hypoxic napA nodules. Unlike the normoxic napA bacteroids, the hypoxic napA bacteroids exhibited some nitrate reductase activity, although at a rate sixfold lower than that in the hypoxic WT bacteroids (Fig. 4b). Likewise, less nitrite accumulation was observed in the hypoxic napA nodules compared to the WT nodules (Fig. 3b). Observation of the EPR spectrum from the hypoxic napA nodules showed that the LbNO signal was half the magnitude of the WT signal (Fig. 1a iv, b). Similarly, the difference between the hypoxic napA reduced and nitrosylated spectra was larger than that between the WT spectra (Fig. 2d), corresponding to less LbNO. Calculating the proportion of LbNO from the absorption spectra showed that the level of LbNO in the napA nodules was half that in the WT nodules, at approximately $30 \%$ (Fig. 3a).

\section{The contribution of NirK to LbNO formation in hypoxic nodules}

Like nap expression and nitrate reductase activity, nirK expression and nitrite reductase activity were also upregulated in bacteroids from nodules of plants subjected to hypoxic stress. To investigate the contribution of nitrite reduction by NirK to LbNO formation in response to hypoxic stress, bacteroidal nitrite reductase activity and nodular nitrite and $\mathrm{LbNO}$ accumulation were observed in hypoxic nirK nodules. As under normoxic conditions, the levels of bacteroidal nitrite reductase activity and nodular nitrite accumulation in hypoxic nirK nodules were similar to the levels in the hypoxic WT nodules (Figs $4 \mathrm{c}$ and $3 \mathrm{~b}$ ). Correspondingly, the magnitude of the hypoxic nirK EPR LbNO signal (Fig. 1a vi, b) and the differences between the reduced and nitrosylated nirK nodule extract absorption spectra (Fig. 2f) were similar to those observed in the hypoxic WT spectra. Determination of the proportion of LbNO within hypoxic nirK nodules showed that, as in the hypoxic WT nodules, approximately $70 \%$ of the Lb was bound to $\mathrm{NO}^{\bullet}$ (Fig. 3a).

\section{DISCUSSION}

LbNO has previously been identified in nitrate-treated soybean nodules, where its formation has been proposed to inhibit nitrogenase activity through the decrease of available Lb to bind oxygen, thereby limiting oxygen diffusion to the bacteroids (Kanayama \& Yamamoto, 1990b). NO for the formation of LbNO has been proposed to arise from nitrite accumulation within the nodules from nitrate reduction by the plant nitrate reductase (Kanayama \& Yamamoto, 1990a). However, we reasoned that $\mathrm{NO}^{\circ}$ might also be produced through the activities of the B. japonicum denitrifying Nap and NirK enzymes, which can reduce nitrate to nitrite, and nitrite to $\mathrm{NO}^{\circ}$, respectively.

We initially established that there was expression of the nap operon in bacteroids from normoxic nodules, suggesting that the free oxygen concentration within infected nodules was low enough to allow expression of this denitrifying gene. Bacteroids from normoxic nodules were also found to have MV-dependent nitrate reductase activity, suggesting that nitrate reduction by normoxic bacteroids had a role in LbNO formation. Accordingly, analysis of normoxic nodules formed by $B$. japonicum cells lacking a functional napA gene showed that bacteroids from these nodules lacked MVdependent nitrate reductase activity. No nitrite accumulated and no LbNO formed within these nodules. This established that, under normoxic conditions in the presence of nitrate, nitrite produced by the $B$. japonicum periplasmic nitrate reductase is the major source of LbNO within soybean nodules. This is in contrast to the proposal by Kanayama \& Yamamoto (1990a) that LbNO formation within nodules may be caused by nitrite accumulation generated from nitrate reduction by the plant nitrate reductase.

Although we have demonstrated a role for the bacteroidal Nap enzyme in nodular LbNO formation, the role of NirK is less clear. We have confirmed that nirK is expressed in normoxic WT bacteroids. However, normoxic nirK nodules had a WT rate of bacteroidal nitrite reductase activity. This established the presence of an alternative nitrite reductase to NirK that may be upregulated in nirK mutant bacteroids. Whilst the NapA nitrate reductase is a possible candidate for nitrite reduction, we believe this to be unlikely, given that 
detailed studies on the highly similar ( $88 \%)$ NapA of Paracoccus pantotrophus show it to be completely nonreactive towards nitrite (Butler et al., 1999). In B. japonicum, the published genome sequence (Kaneko et al., 2002) reveals the presence of a nirB gene, which may encode a nitrite reductase that reduces nitrite during nitrate assimilation (Lin \& Stewart, 1998). If this enzyme were active in the nirK bacteroids, it might not be expected to contribute to the formation of LbNO from the reduction of nitrite, since an assimilatory nitrite reductase reduces nitrite to ammonium, not to nitric oxide (Lin \& Stewart, 1998), though the release of $\mathrm{NO}^{\circ}$ as a side product of this reaction cannot be excluded. The observation of a WT level of LbNO within normoxic nirK nodules does, however, demonstrate that NirKindependent routes for the reduction of nitrite to $\mathrm{NO}^{\circ}$ are present and that these need not be bacteroidal. For example, nitrite may be reduced to $\mathrm{NO}^{*}$ by the plant nitrate reductase (Yamasaki et al., 1999) or by non-enzymic reduction (Yamasaki \& Sakihama, 2000), which may occur in nodules, due to the acidic environment within symbiosomes (Blumwald et al., 1985) and the presence of high levels of reductant required for nitrogen fixation (Haaker, 1988).

In the transition from normoxia to hypoxia, the levels of nitrite and LbNO within WT nodules both underwent a 2.5fold increase, suggesting a correlation between nitrite accumulation within nodules and LbNO formation. In addition, nap and nirK expression and nitrate and nitrite reductase activities were upregulated in bacteroids from these nodules, suggesting that the denitrification system in B. japonicum bacteroids may have a role in this hypoxiainduced increase of LbNO. In contrast to normoxic napA nodules, some bacteroidal MV-dependent nitrate reductase activity and nodular nitrite and LbNO accumulation were observed in the napA hypoxic nodules, although at levels significantly lower than those in the WT hypoxic nodules. A possible candidate for this bacteroidal nitrate reductase activity is an assimilatory nitrate reductase which reduces nitrate to nitrite for biosynthetic purposes (Lin \& Stewart, 1998). Inspection of the B. japonicum complete genome sequence (Kaneko et al., 2002) does indeed reveal the presence of an ORF, nasA, that may encode an assimilatory nitrate reductase. This enzyme may be responsible for the nitrite accumulation observed in the hypoxic napA nodules. Further investigation is required as to why such an enzyme would only be expressed under anaerobic conditions in the nodule, but we note that in Sinorhizobium medicae, the nasA gene (termed narB in this species) is under the control of ActR, which regulates nitrogen metabolism in response to low pH and micro-aerobioses (Fenner et al., 2004). NasA is predicted to have a cytoplasmic location. Since reduced benzyl viologen (BV) is more membrane-permeant than reduced MV, we also attempted to assay the enzyme with this electron donor; however, no activity was detected. Reduced BV is not as strong a reducing agent as reduced MV. Cytoplasmic bacterial assimilatory nitrate reductase operates in a very low-potential domain (Jepson et al., 2004), and although reduced MV is less membrane-permeant than reduced $\mathrm{BV}$, its stronger reducing power may still make it a more effective electron donor to cytoplasmic assimilatory nitrate and nitrite reductases.

Although the bacteroidal nitrate reductase activity in the hypoxic napA nodules may be responsible for nitrite accumulation, nitrate reduction by the plant nitrate reductase may also contribute, since activity of this enzyme is upregulated under hypoxia (Allegre et al., 2004; Glaab \& Kaiser, 1993). Furthermore, nitrate has been shown to be a precursor of $\mathrm{NO}^{\circ}$ in anoxic maize cell-suspension cultures (Dordas et al., 2004). In addition, it has recently been demonstrated that mitochondria from plant root tissue are also able to reduce nitrite to $\mathrm{NO}^{*}$, with the highest rates of NO production observed under hypoxia/anoxia (Gupta et al., 2005). However, whether the plant nitrate reductase or root mitochondria contribute to LbNO formation in soybean nodules in response to hypoxia has yet to be addressed.

Under hypoxic stress, nitrite accumulated in the napA nodules to $25 \%$ of that in the WT nodules; however, nitrosylation of the Lb was $50 \%$ of that in the WT nodules. This suggests the presence of a nitrite-independent source of $\mathrm{NO}^{\circ}$ within nodules, possibly arising from the action of a plant NOS which uses arginine, oxygen and NADPH to produce $\mathrm{NO}^{\circ}$ and citrulline (Lamotte et al., 2005). Argininedependent NOS activity has been detected in nodules of Lupinus albus (Cueto et al., 1996), and the Arabidopsis AtNOS1 protein has been implicated in $\mathrm{NO}^{\circ}$ synthesis (Guo et al., 2003). Whether a plant NOS is present and functional in soybean nodules is unknown, although recent evidence suggests that $\mathrm{NO}^{\circ}$ production by NOS contributes to the $\mathrm{NO}^{*}$ in Medicago truncatula nodules (Baudouin et al., 2006).

As in the normoxic nirK nodules, the levels of bacteroidal nitrite reductase activity and nodular nitrite and LbNO accumulation in the hypoxic nirK nodules were similar to those observed in the hypoxic WT nodules. In a similar manner to the normoxic nodules, nitrite reduction by the plant nitrate reductase and non-enzymic nitrite reduction may be responsible for the nitrite-derived LbNO formation observed in hypoxic nirK nodules, and NirB could be responsible for the bacteroidal nitrite reductase activity detected in the nirK mutant. Thus, on the basis of these data, no role can be definitively assigned to NirK in LbNO formation in response to hypoxic stress.

In conclusion, our results clearly demonstrate that the process of nitrate reduction by Nap in bacteroids makes an important contribution to the formation of LbNO in soybean nodules. The relevance of this contribution in nodules from soybean plants subjected to physiological hypoxic conditions, such as drought and flooding, is currently being investigated.

\section{ACKNOWLEDGEMENTS}

G. E. M. was supported by the studentship grant BBSSP200310318 provided by the Biotechnology and Biological Sciences Research 
Council to D. J. R. We thank H.-M. Fischer, Mikrobiologisches Institut, Eidgenossische Technische Hochschule, Zurich, for the gift of the plasmid pSUP3535. We also thank E. Raven, University of Leicester, for providing the $\mathrm{Lb}$ expression strain. This work was supported by the grant Aquarhiz INCO-CT2004-509115. Support from the Junta de Andalucía (PAI/CVI-275) is also acknowledged, as is the financial support from the Royal Society (Grant 2004/R1-EU) for the CSICUEA cooperation agreement and the COST Action 856.

\section{REFERENCES}

Allegre, A., Silvestre, J., Morard, P., Kallerhoff, J. \& Pinelli, E. (2004). Nitrate reductase regulation in tomato roots by exogenous nitrate: a possible role in tolerance to long-term root anoxia. J Exp Bot 55, 2625-2634.

Appleby, C. A. (1992). The origin and functions of hemoglobin in plants. Sci Progress 76, 365-398.

Baudouin, E., Pleuchot, L., Engler, G., Pauly, N. \& Puppo, A. (2006). Nitric oxide is formed in Medicago truncatula-Sinorhizobium meliloti functional nodules. Mol Plant Microbe Interact 19, 970-975.

Blumwald, E., Fortin, M. G., Rea, P. A., Verma, D. P. A. \& Roole, R. J. (1985). Presence of host-plasma membrane type $\mathrm{H}^{+}$-ATPase in the membrane envelope enclosing the bacteroids in soybean root nodules. Plant Physiol 78, 665-672.

Butler, C. S., Charnock, J. M., Bennett, B., Sears, H. J., Reilly, A. J., Ferguson, S. J., Garner, C. D., Lowe, D. J., Thomson, A. J. \& other authors (1999). Models for molybdenum co-ordination during the catalytic cycle of periplasmic nitrate reductase from Paracoccus denitrificans derived from EPR and EXAFS spectroscopy. Biochemistry 38, 9000-9012.

Cueto, M., Hernández-Perera, O., Martín, R., Bentura, M. L., Rodigo, J., Lamas, S. \& Golvano, M. P. (1996). Presence of nitric oxide synthase activity in roots and nodules of Lupinus albus. FEBS Lett 398, 159-164.

Delgado, M. J., Bedmar, E. J. \& Downie, J. A. (1998). Genes involved in the formation and assembly of rhizobial cytochromes and their role in symbiotic nitrogen fixation. Adv Microbial Phys 40, 191-231.

Delgado, M. J., Bonnard, N., Tresierra-Ayala, A., Bedmar, E. J. \& Müller, P. (2003). The Bradyrhizobium japonicum napEDABC genes encoding the periplasmic nitrate reductase are essential for nitrate respiration. Microbiology 149, 3395-3403.

Dordas, C., Hasinoff, B. B., Igamberdiev, A. U., Manach, N., Rivoal, J. \& Hill, R. D. (2003). Expression of a stress-induced hemoglobin affects NO levels produced by alfalfa root cultures under hypoxic stress. Plant J 35, 763-770.

Dordas, C., Hasinoff, B. B., Rivoal, J. \& Hill, R. D. (2004). Class-1 hemoglobins, nitrate and NO levels in anoxic maize cell-suspension cultures. Planta 219, 66-72.

Fenner, B. J., Tiwari, R. P., Reeve, W. G., Dilworth, M. J. \& Glenn, A. R. (2004). Sinorhizobium medicae gene whose regulation involves the ActS and/or ActR signal transduction proteins. FEMS Microbiol Lett 236, 21-31.

Glaab, J. \& Kaiser, W. M. (1993). Rapid modulation of nitrate reductase in pea roots. Planta 191, 173-179.

Guo, F.-Q., Okamoto, M. \& Crawford, N. M. (2003). Identification of a plant nitric oxide synthase gene involved in hormonal signalling. Science 302, 100-103.

Gupta, K. J., Stoimenova, M. \& Kaiser, W. M. (2005). In higher plants, only root mitochondria, but not leaf mitochondria reduce nitrite to NO, in vitro and in situ. J Exp Bot 56, 2601-2609.
Haaker, H. (1988). Biochemistry and physiology of nitrogen fixation. BioEssays 9, 112-117.

Herold, S. \& Puppo, A. (2005). Oxyleghaemoglobin scavenges nitrogen monoxide and peroxynitrite: a possible role in functioning nodules? J Biol Inorg Chem 10, 935-945.

Jepson, B., Anderson, L., Rubio, L., Taylor, C., Butler, C., Herrero, A., Flores, E., Butt, J. N. \& Richardson, D. J. (2004). Tuning a nitrate reductase for function: the first spectropotentiometric characterization of a bacterial assimilatory nitrate reductase reveals novel redox properties. J Biol Chem 279, 32212-32218.

Jones, D. K., Badii, R., Rosell, F. I. \& Lloyd, E. (1998). Bacterial expression and spectroscopic characterization of soybean leghaemoglobin a. Biochem J 330, 983-988.

Kanayama, Y. \& Yamamoto, Y. (1990a). Inhibition of nitrogen fixation in soybean plants supplied with nitrate II. Accumulation and properties of nitrosylleghaemoglobin in nodules. Plant Cell Physiol 31, 207-214

Kanayama, Y. \& Yamamoto, Y. (1990b). Inhibition of nitrogen fixation in soybean plants supplied with nitrate III. Kinetics of the formation of nitrosylleghaemoglobin and of the inhibition of formation of oxyleghaemoglobin. Plant Cell Physiol 31, 603-608.

Kanayama, Y., Watanabe, I. \& Yamamoto, Y. (1990). Inhibition of nitrogen fixation in soybean plants supplied with nitrate I. Nitrite accumulation and formation of nitrosylleghaemoglobin in nodules. Plant Cell Physiol 31, 341-346.

Kaneko, T., Nakamura, Y., Sato, S., Minamisawa, K., Ughiuma, T., Sasamoto, S., Watanabe, A., Idesawa, K., Iriguchi, M. \& other authors (2002). Complete genomic sequence of nitrogen-fixing symbiotic bacterium Bradyrhizobium japonicum USDA110. DNA Res 9, 189-197.

Lamotte, O., Courtois, C., Barnavon, L., Pugin, A. \& Wendehenne, D. (2005). Nitric oxide in plants: the biosynthesis and cell signalling properties of a fascinating molecule. Planta 221, 1-4.

Lin, J. T. \& Stewart, V. (1998). Nitrate assimilation by bacteria. $A d v$ Microb Physiol 39, 1-30.

Linkemer, G., Board, J. E. \& Musgrave, M. E. (1998). Waterlogging effects on growth and yield components in late-planted soybean. Crop Sci 38, 1576-1584.

Mathieu, C., Moreau, S., Frendo, P., Puppo, A. \& Davies, M. J. (1998). Direct detection of radicals in intact soybean nodules: presence of nitric oxide-leghaemoglobin complexes. Free Radic Biol Med 24, 1242-1249.

Mesa, S., Velasco, L., Manzanera, M. E., Delgado, M. J. \& Bedmar, E. J. (2002). Characterisation of the norBCQD genes, encoding nitric oxide reductase, in the nitrogen fixing bacterium Bradyrhizobium japonicum. Microbiology 148, 3553-3560.

Mesa, S., Bedmar, E. J., Chanfon, A., Hennecke, H. \& Fischer, H.-M. (2003). Bradyrhizobium japonicum $\mathrm{NnrR}$, a denitrification regulator, expands the FixLJ-FixK2 regulatory cascade. J Bacteriol 185, 3978-3982.

Mesa, S., de Dios Alché, J., Bedmar, E. J. \& Delgado, M. J. (2004). Expression of the nir, nor and nos denitrification genes from Bradyrhizobium japonicum in soybean root nodules. Physiol Plant 120, 205-211.

Miller, J. H. (1972). Experiments in Molecular Genetics. Cold Spring Harbor, NY: Cold Spring Harbor Laboratory.

Minchin, F. R. (1997). Regulation of oxygen diffusion in legume nodules. Soil Biol Biochem 29, 881-888.

Nicholas, D. J. D. \& Nason, A. (1957). Determination of nitrate and nitrite. In Methods in Enzymology, pp. 981-984. Edited by S. P. Colowick \& N. O. Kaplan. New York: Academic Press.

Preisig, O., Zufferey, R., Thöny-Meyer, L., Appleby, C. A. \& Hennecke, H. (1996). A high-affinity $c b b_{3}$-type cytochrome oxidase 
terminates the symbiosis-specific respiratory chain of Bradyrhizobium japonicum. J Bacteriol 178, 1532-1538.

Regensburger, B. \& Hennecke, H. (1983). RNA polymerase from Rhizobium japonicum. Arch Microbiol 135, 103-109.

Rigaud, J. \& Puppo, A. (1975). Indole-3-acetic acid catabolism by soybean bacteroids. J Gen Microbiol 88, 223-228.

Robles, E. F., Sánchez, C., Delgado, M. J. \& Bedmar, E. J. (2006). The Bradyrhizobium japonicum napEDABC genes are controlled by the FixLJ-FixK2-NnrR regulatory cascade. Biochem Soc Trans 34, 108-110.

Velasco, L., Mesa, S., Delgado, M. J. \& Bedmar, E. J. (2001). Characterization of the nirK gene encoding the respiratory, $\mathrm{Cu}$-containing nitrite reductase of Bradyrhizobium japonicum. Biochim Biophys Acta $1521,130-134$.
Velasco, L., Mesa, S., Xu, C., Delgado, M. J. \& Bedmar, E. J. (2004). Molecular characterization of nosRZDFYLX genes coding for denitrifying nitrous oxide reductase of Bradyrhizobium japonicum. Antonie van Leeuwenhoek 85, 229-235.

Yamasaki, H. \& Sakihama, S. (2000). Simultaneous production of nitric oxide and peroxynitrite by plant nitrate reductase: in vitro evidence for the NR-dependent formation of active nitrogen species. FEBS Lett 468, 89-92.

Yamasaki, H., Sakihama, Y. \& Takahashi, S. (1999). An alternative pathway for nitric oxide production in plants: new features of an old enzyme. Trends Plant Sci 4, 128-129.

Edited by: M. F. Hynes 